\title{
Factores asociados con la mortalidad en el adulto mayor con choque séptico
}

Factors associated with mortality in the elderly adult with septic shock

Fatores associados à mortalidade no idoso com choque séptico

Eduardo Alberto González Escudero, ${ }^{*}$ Jesús Salvador Sánchez Díaz, ${ }^{*}$ Armando Solórzano Guerra, ${ }^{*}$
Karla Gabriela Peniche Moguel, ${ }^{\star}$ Josué Eli Villegas Domínguez, ${ }^{*}$ María Verónica Calyeca Sánchez ${ }^{*}$

\section{RESUMEN}

Introducción: Se estima que para el año 2050, a nivel mundial y por primera vez en la historia, la población anciana será mayor que la población joven. Con respecto a este grupo de edad, se calcula que la incidencia de choque séptico es 13 veces más común en los pacientes mayores de 65 años, por lo que conocer los factores de riesgo asociados con mortalidad en esta población es indispensable para la terapéutica.

Material y métodos: Estudio ambispectivo, longitudinal, descriptivo y analítico en el que se incluyeron a pacientes con diagnóstico de choque séptico según el consenso Sepsis-3 ingresados a la Unidad de Cuidados Intensivos del primero de junio de 2015 al 31 de junio de 2019. Se clasificaron los grupos en: $<65$ y $>65$ años, y se incluyeron variables demográficas, hemodinámicas (índice de choque modificado, $\mathrm{SvcO}_{2}, \Delta \mathrm{p}(\mathrm{v}-\mathrm{a}) \mathrm{CO}_{2} / \Delta \mathrm{p}(\mathrm{a}-\mathrm{v}) \mathrm{O}_{2}$, BNP, vasopresor), respiratorias $\left(\mathrm{PaO}_{2} / \mathrm{FiO}_{2}\right)$, metabólicas $\left(\mathrm{pH}, \mathrm{HCO}_{3}-\mathrm{DB}\right)$ y renales, para compararlas entre ambos grupos. El grupo > 65 años se subclasificó de acuerdo con el desenlace en vivos y no vivos, para identificar a las variables con valor estadístico para el mismo; se utilizó estadística descriptiva, $\chi^{2}$ y prueba exacta de Fisher y $t$ de Student y $U$ de Mann-Whitney de acuerdo con el caso. Se consideró significancia un valor de $\mathrm{p} \leq 0.05$ y se empleó el programa SPSS en su versión 20.

Resultados: Un total de 118 pacientes cumplieron con los criterios de inclusión. De ellos, 78 pacientes se designaron al grupo $<65$ años y 40 pacientes al grupo $>65$ años. La mortalidad en el grupo > 65 años fue de $67.5 \%$ en comparación con $36 \%$ en el grupo $<65$ años; con $p=0.001$. En el grupo $>65$ años, 27 pacientes fallecieron. Las variables hemodinámicas y respiratorias no tuvieron significancia estadística, a diferencia del $\mathrm{pH}(\mathrm{p}=0.037)$, base $(p=0.041)$ y norepinefrina $(p=0.03)$.

Conclusión: Los pacientes > 65 años de edad tienen una mayor gravedad y comorbilidad respecto a los pacientes más jóvenes. Las variables hemodinámicas y respiratorias no influyen en el desenlace; la acidosis metabólica grave es un factor de riesgo para mortalidad en los pacientes > 65 años.

Palabras clave: Choque séptico, factores de riesgo, mortalidad.

\section{ABSTRACT}

Introduction: By 2050 the elderly population worldwide for the first time in history will be greater than the young population. The incidence of septic shock is 13 times more common in patients > 65 years, knowing the risk factors associated with mortality in this population is essential for therapeutics.

Material and methods: Ambispective, longitudinal, descriptive, analytical study. Patients with a diagnosis of septic shock according to the consensus Sepsis-3 admitted to the ICU from June 1, 2015 to June 31, 2019. They were classified in the groups: 65 years, demographic, hemodynamic variables were included (index modified shock, $\mathrm{SvcO}_{2}, \Delta p$ (va) $\mathrm{CO}_{2} / \Delta p$ (av) $\mathrm{O}_{2}, \mathrm{BNP}$, vasopressor), respiratory $\left(\mathrm{PaO}_{2} / \mathrm{FiO}_{2}\right)$, metabolic $\left(\mathrm{pH}, \mathrm{HCO}_{3}-\mathrm{DB}\right)$ and renal to compare them between both groups; the group $>65$ years was subclassified according to the outcome in living and non-living to identify the variables with statistical value for it; descriptive statistics, $\chi^{2}$ and exact test of Fisher and t-Student and U-MannWhitney were used according to the case. A value of $p \leq 0.05$ was considered significance and the program was used SPSS in version 20.

Results: 118 patients met the inclusion criteria. 78 patients in the group 65 years. Mortality in > 65 years was $67.5 \%$ versus $36 \%$ in 65 years, 27 patients died. The hemodynamic and respiratory variables had no statistical significance; unlike $\mathrm{pH}(p=0.037)$, base $(p=0.041)$ and norepinephrine $(p=0.03)$.

Conclusion: Patients $>65$ years of age have greater severity and comorbidity

* Hospital de Especialidades Núm. 14. Centro Médico Nacional «Adolfo Ruiz Cortines», Instituto Mexicano del Seguro Social. Veracruz, Veracruz.

‡ Facultad de Medicina de la Universidad del Valle de México (UVM), Campus Veracruz. Veracruz, México.

Recepción: 13/08/2019. Aceptación: 04/02/2020.

www.medigraphic.com/medicinacritica compared to younger patients. The hemodynamic and respiratory variables do not influence the outcome; severe metabolic acidosis is a risk factor for mortality in patients $>65$ years.

Keywords: Septic shock, risk factors, mortality.

\section{RESUMO}

Introdução: Até 2050, a população idosa em todo o mundo pela primeira vez na história será maior que a população jovem. A incidência de choque séptico é 13 vezes mais comum em pacientes > 65 anos, sabendo que os fatores de risco associados à mortalidade nessa população são essenciais para a terapêutica.

Material e métodos: Estudo ambispectico, longitudinal, descritivo e analítico. Pacientes com diagnóstico de choque séptico segundo o consenso Sepsis-3 admitidos na UTI de $1^{\circ}$ de junho de 2015 a 31 de junho de 2019. Eles foram classificados nos grupos: $<65$ e > 65 anos. Foram incluídas variáveis demográficas, hemodinâmicas (índice de choque modificado, $\mathrm{SvcO}_{2}, \Delta p(v-a)$ $\mathrm{CO}_{2} / \mathrm{Ap}(\mathrm{a}-\mathrm{v}) \mathrm{O}_{2}, \mathrm{BNP}$, vasopressor), respiratórios $\left(\mathrm{PaO}_{2} / \mathrm{FiO}_{2}\right)$, metabólicos $\left(\mathrm{pH}, \mathrm{HCO}_{3}-\mathrm{DB}\right)$ e renais para compará-los entre os dois grupos; o grupo > 65 anos foi subclassificado de acordo com o desfecho em sobreviver e não sobreviver para identificar as variáveis com valor estatístico para ele. Foram utilizados estatística descritiva, $\chi^{2}$ e teste exato de Fisher e T-Student e U-Mann-Whitney de acordo com o caso. Um valor de $p \leq 0.05$ foi considerado significativo, foi utilizado o SPPSS20.

Resultados: 118 pacientes preencheram os critérios de inclusão. 78 pacientes no grupo < 65 anos e 40 pacientes no grupo $>65$ anos. A mortalidade em $>$ 65 anos foi de $67.5 \%$ versus $36 \%$ em $<65$ anos com $p=0.001$. No grupo $>65$ anos, 27 pacientes morreram. As variáveis hemodinâmicas e respiratórias não apresentaram significância estatística; diferentemente do $\mathrm{pH}(\mathrm{p}=0.037)$, base $(p=0.041)$ e noradrenalina $(p=0.03)$.

Conclusão: Pacientes com idade > 65 anos apresentam maior gravidade e comorbidade que pacientes mais jovens. As variáveis hemodinâmicas e respiratórias não influenciam o resultado; $A$ acidose metabólica grave é um fator de risco para mortalidade em pacientes $>65$ anos.

Palavras-chave: Choque séptico, fatores de risco, mortalidade.

\section{INTRODUCCION}

Para el año 2050, por primera vez y a nivel mundial en la historia de la humanidad, la población anciana será mayor que la población joven. Por un lado y siguiendo esta tendencia, el incremento en la esperanza de vida conlleva a que en la actualidad en las Unidades de Cuidados Intensivos (UCl) sea cada vez más frecuente encontrar pacientes ancianos con choque séptico;,1,2 de hecho, la incidencia de choque séptico es 13 veces más común en los pacientes mayores de 65 años. Por otro lado, la mitad de los días de estancia en la UCI corresponde a este grupo de pacientes, lo que implica que se utilicen más recursos con la longevidad. ${ }^{3-5}$ Por tanto, la edad es identificada como un factor de riesgo independiente asociado con mortalidad en las diferentes poblaciones de pacientes críticamente enfermos y, en este sentido, el choque séptico no es la excepción. ${ }^{6}$

A pesar de todo el cúmulo de avances que existen sobre el monitoreo de los pacientes con choque 
séptico y la disminución del tiempo para cumplir tempranamente los bundles de la campaña «Sobreviviendo a la sepsis", el choque séptico sigue ocupando un decoroso segundo lugar como causa de muerte no coronaria en las Unidades de Cuidados Intensivos, alcanzando una mortalidad hospitalaria de 18 al $35 \% .{ }^{7} \mathrm{~A}$ nivel mundial, 14 millones de pacientes sobrevivirán al choque séptico, pero la gran mayoría presentará incapacidad a largo plazo, deterioro de la calidad de vida por la morbilidad y sólo la mitad de los pacientes que padecieron choque séptico tendrá una recuperación completa. ${ }^{8}$ Una constante en los estudios que abordan este tema es que la mortalidad posterior al alta hospitalaria es significativamente mayor en pacientes $>65$ años (27 contra 8\%). ${ }^{9,10}$ En este contexto, es importante conocer la epidemiología y los factores de riesgo que influyen en los resultados de los pacientes vulnerables, en este caso, la población con edad igual o mayor a 65 años, ya que con ello se podrá orientar las decisiones de tratamiento y evaluar las escalas pronósticas.

La edad avanzada se asocia con una mayor vulnerabilidad a la infección, debido principalmente a los cambios fisiológicos relacionados con el envejecimiento, así como con una mayor frecuencia de enfermedades crónicas, polifarmacia, y dispositivos y contacto con el sistema hospitalario. ${ }^{11} \mathrm{~A}$ nivel celular, el envejecimiento se puede definir como un deterioro progresivo de la estructura y función que ocurre con el tiempo. Los factores que conducen al envejecimiento primario son poco entendidos, sin embargo, se sabe que la interacción entre genética y estrés oxidativo juegan un papel importante. ${ }^{12}$ Martin y colaboradores documentaron un incremento del riesgo de infección por Gram-negativos en pacientes ancianos en comparación con pacientes jóvenes con un RR de 1.33 (IC 95\%; 1.27-1.35); asimismo, encontraron que la probabilidad de infecciones respiratorias (RR 1.29 [IC 95\%; 1.25-1.33]) y genitourinarias (RR 1.38 [IC 95\%; 1.32-1.44]) es más grande en los pacientes de mayor edad. ${ }^{13}$ Una evaluación cuidadosa de los signos del síndrome de respuesta inflamatoria sistémica (SRIS) es determinante en los pacientes ancianos; ${ }^{14}$ de hecho, hasta $15 \%$ de los pacientes $>65$ años no presentará fiebre. ${ }^{15}$ Muy importante son las expresiones clínicas inespecíficas de infección en los pacientes de edad avanzada; entre las más comunes se puede incluir delirio, debilidad, anorexia, malestar general, incontinencia urinaria o caídas. ${ }^{16}$ Las infecciones en el paciente anciano presentan una menor expresión clínica y suelen desarrollarse de forma atípica, con una sintomatología más larvada que en los pacientes jóvenes. De esta manera, no es raro que se produzca un retraso diagnóstico que, junto con la mayor agresividad de las infecciones debido a los diferentes factores anteriormente citados, provoque un incremento de la mor- bimortalidad y empeore el pronóstico global respecto al resto de la población. ${ }^{17}$

Conforme avanza la edad de un individuo, ocurrirá un envejecimiento primario, el cual es el que condiciona el deterioro en la estructura y función celular, independientemente de si se está enfermo o sano. Las consecuencias del envejecimiento primario incluyen alteraciones en la función cardiaca, pulmonar, renal y metabólica. ${ }^{18}$ Hoy en día, reconocemos a la edad como uno de los principales factores de riesgo para enfermedades cardiovasculares, representando $40 \%$ de las muertes en pacientes mayores de 65 años. La disminución de la reserva cardiaca puede no afectar el funcionamiento diario de un individuo "sano", pero en condiciones de estrés, como en el choque séptico, la baja reserva miocárdica se vuelve evidente. Con el envejecimiento, existe una disminución progresiva en el número de miocitos y un incremento del contenido de colágeno en el miocardio. El tejido autónomo es remplazado por tejido conectivo y grasa, y finalmente la fibrosis causa alteraciones de la conducción y de la estructura miocárdica. ${ }^{19,20}$

La disminución de la función pulmonar en los ancianos es el resultado de los cambios disminuidos en la pared torácica y el pulmón; su capacidad inspiratoria y espiratoria se verá afectada en $50 \%$. Asimismo, a partir de los 30 años, la presión arterial de oxígeno caerá 0.3 $\mathrm{mmHg}$ por año. La respuesta ventilatoria a la hipoxia e hipercapnia disminuyen en 50 y $40 \%$, respectivamente ${ }^{21}$ entre los 25 y 85 años de edad, $40 \%$ de las nefronas se vuelven escleróticas, y el flujo sanguíneo renal disminuye, aproximadamente, $50 \%$. Funcionalmente, a la edad de 80 años, hay un declive en la tasa de filtración glomerular (TFG) de 45\%, 22 y la función metabólica cambia con el envejecimiento celular: se incrementa la grasa corporal y se disminuye la masa muscular magra hasta $40 \%$. El gasto energético y el consumo de oxígeno diario caerán en $20 \%{ }^{23}$

Por todo lo anteriormente mencionado, la edad queda definida como un factor determinante de los resultados en la UCl. Por ello, es importante conocer los factores de riesgos asociados con mortalidad en pacientes ancianos con choque séptico, para así seleccionar la mejor intervención.

Objetivo: Identificar los factores de riesgo asociados con mortalidad en el paciente adulto mayor con choque séptico.

\section{MATERIAL Y MÉTODOS}

Bajo la autorización del Comité de Investigación y Ética del hospital, se realizó un estudio de riesgo mínimo y de no intervención: ambispectivo, longitudinal, descriptivo y analítico que incluyó pacientes con diagnóstico de choque séptico, de acuerdo con el tercer Consenso Internacional de Sepsis y Choque Séptico (Sepsis-3) que 
fueron ingresados en la $\mathrm{UCl}$ en el periodo comprendido entre el primero de junio de 2015 al 31 de junio de 2019.

Criterios de inclusión: pacientes mayores de 18 años de edad con diagnóstico de choque séptico según el tercer Consenso Internacional de Sepsis y Choque Séptico (Sepsis-3).

Criterios de exclusión: pacientes menores de 18 años con registro de variables incompleto.

Criterios de eliminación: pacientes que concluyan su tratamiento en otra terapia intensiva, mujeres embarazadas y aquéllos con orden de no reanimación o de máximo alcance terapéutico.

Procedimiento: después del ingreso a la $\mathrm{UCI}$, se agregaron al estudio los pacientes que cumplieron con los criterios de inclusión y se obtuvieron las variables necesarias para el estudio. La terapéutica empleada para el paciente no estuvo influenciada por la recolección de datos, más bien quedó a criterio del médico intensivista de turno. De acuerdo con la edad, se clasificaron los pacientes en dos grupos: en > 65 años y < 65 años; posteriormente, se compararon las variables entre ambos grupos. Los datos se vaciaron en una hoja de Microsoft Excel.

Análisis estadístico: los datos se analizaron mediante estadística descriptiva para determinar las características generales de la población. Las variables categóricas se analizaron con $\chi^{2}$ y la prueba exacta de Fisher; las variables continuas con prueba de $t$ de Student. Se usó $U$ de Mann-Whitney para variables de distribución anormal. Se consideró significancia estadística un valor de $p<0.05$. El análisis estadístico se realizó con el programa SPSS ${ }^{\mathrm{TM}}$ versión 20.

\section{RESULTADOS}

Se reclutaron 118 pacientes, que cumplieron con los criterios de inclusión en el periodo considerado. Se clasificaron de acuerdo con la edad en dos grupos: $<65$ años (media de 45 años) y > 65 años (media de 72 años). En el primer grupo se incluyeron 78 pacientes, de los cuales $50.6 \%$ fue del género femenino, en tanto que en el segundo grupo la población total fue de 40 pacientes, donde $60 \%$ del total lo conformaron mujeres.

La media del índice de masa corporal (IMC) fue de 28.59 para < 65 años y 27.13 para $>65$ años, sin que esto mostrara relevancia estadística. El puntaje de severidad de SAPS (Simplified Acute Physiology Score) II mostró una mayor gravedad en el grupo $>65$ años; esto con un valor de $71 \pm 10.3$ y de $66 \pm 12.5$ en el grupo $<65$ años $(p=0.001)$. La mortalidad fue mayor en los pacientes $>65$ años con choque séptico con $67.5 \%$, y de $36 \%$ en los $<65$ años $(p=0.001)$. Dentro de las comorbilidades, destaca la diabetes mellitus tipo 2 (DM2) y la hipertensión arterial sistémica (HAS) como las más frecuentes en ambos grupos, sin embargo, la cardiopatía (isquémica y no isquémica) en el grupo > 65 años tuvo una significancia estadística comparada con el grupo < 65 años $(p=0.04)$. El origen abdominal del choque séptico fue el más frecuente en ambos grupos, con 39.7\% (< 65 años) y $62.5 \%$ (> 65 años). Para el grupo < 65 años, el servicio que contribuyó mayormente a los ingresos a la $\mathrm{UCl}$ fue el de Medicina de Urgencias y el de Cirugía, con $43.6 \%$ de los casos cada uno, mientras que en el grupo $>65$ años el Servicio de Cirugía aportó $67.5 \%$. El tratamiento más frecuente fue el quirúrgico, con $67.9 \%$ para los $<65$ y de $72.5 \%$ para el grupo $>65$ años, respectivamente. La infección comunitaria fue de $71.8 \%$ para los menores de 65 años y de $60 \%$ para los mayores de 65 años. Variables como no estancia en la UCl, estancia en la $\mathrm{UCl}$, índice de choque modificado, $\mathrm{SvCO}_{2}$ (índice de choque modificado, saturación venosa central de oxígeno),) $\Delta \mathrm{p}(\mathrm{v}-\mathrm{a})$ (delta de presión venoarterial de dióxido de carbono), $\mathrm{CO}_{2} / \Delta \mathrm{p}(\mathrm{a}-\mathrm{v}) \mathrm{O}_{2}$ (delta de presión venoarterial de dióxido de carbono), BNP (B-type Natriuretic Peptide), $\mathrm{PaO}_{2} / \mathrm{FiO}_{2}$ (presión arterial de oxígeno/fracción inspiratoria de oxígeno), $\mathrm{VM}$, creatinina, $\mathrm{HCO}_{3}$ ( (bicarbonato), base, albúmina, balance de líquidos y TRRLC (terapia de remplazo renal lenta continua) no tuvieron diferencia estadísticamente significativa (Tabla 1).

A su vez, al grupo > 65 años de edad, de acuerdo con el desenlace presentado en la $\mathrm{UCl}$, se subclasificó en: vivos y no vivos, con 13 pacientes y 27 pacientes, respectivamente. Las variables analizadas fueron la edad con una media de 74 años para los vivos y 71 años para los no vivos. En el primer grupo, 53.8\% fue del género femenino, mientras que en el segundo grupo fue $63 \%$. La media del índice de masa corporal (IMC) fue de 28 para vivos y 26.6 para los no vivos sin relevancia estadística. El puntaje de severidad SAPS II mostró en el grupo de los vivos un valor de $74.5 \pm 11.9$ y de $70.5 \pm 12.5$ en el grupo de no vivos. Referente a comorbilidades, no existió relevancia estadística; el origen abdominal del choque séptico fue el más frecuente en ambos grupos sin diferencia estadística; el Servicio de Cirugía fue el lugar de donde mayormente procedieron los pacientes; el tratamiento quirúrgico fue el más frecuente, y la infección comunitaria fue la de mayor incidencia en ambos grupos.

Variables como estancia no $\mathrm{UCl}$, estancia en $\mathrm{UCI}$, $\mathrm{SvCO}_{2}$ (índice de choque modificado, saturación venosa central de oxígeno),) $\Delta \mathrm{p}(\mathrm{v}-\mathrm{a})$ (delta de presión venoarterial de dióxido de carbono); $\mathrm{CO}_{2} / \Delta \mathrm{p}(\mathrm{a}-\mathrm{v}) \mathrm{O}_{2}$ (delta de presión venoarterial de dióxido de carbono), BNP (Btype Natriuretic Peptide), $\mathrm{PaO}_{2} / \mathrm{FiO}_{2}$ (presión arterial de oxígeno/fracción inspiratoria de oxígeno), VM, creatinina, $\mathrm{HCO}_{3}-$, albúmina, balance de líquidos y TRRLC (terapia de remplazo renal lenta continua) no tuvieron diferencia estadísticamente significativa, a diferencia de las variables: $\mathrm{pH}(\mathrm{p}=0.037)$, base $(\mathrm{p}=0.041)$ y norepinefrina $(p=0.03)$ (Tabla 2). 


\section{DISCUSIÓN}

El tratamiento del choque séptico tiene enormes implicaciones financieras, de hecho, más de la mitad del gasto es atribuible a la atención de pacientes > 65 años. Por si esto fuera poco, se predice que la frecuencia del choque séptico aumentará en más de $5 \%$ de forma paralela al incremento de la población anciana. ${ }^{24,25}$ Esto ha llevado a un intenso debate sobre si es necesario racionar los recursos, reevaluar los criterios de admisión a la $\mathrm{UCl}$ y si habrá de suspenderse el tratamiento a pacientes de edad avanzada. Al respecto, negar la ad- misión al adulto mayor a la UCI tiene pocos seguidores, pero, al ingresar, el tratamiento insuficiente únicamente por la edad es muy controvertido. Por lo tanto, la edad por sí sola no debe ser la razón para negar la admisión o el tratamiento óptimo en el paciente adulto mayor con choque séptico. ${ }^{26,27}$

Nuestro estudio muestra que la mortalidad es mayor en los pacientes con choque séptico > 65 años, con $67.5 \%$, lo cual no difiere de la literatura mundial que reporta alrededor de $60 \%$ y cerca de $80 \%$ en los $>80$ años, ${ }^{28} \mathrm{si}$ bien en los pacientes $<65$ años observamos mortalidad de $36 \%$. Otros estudios han evaluado la mortalidad en

Tabla 1: Análisis bivariado entre los grupos $<65$ y $>65$ años de edad.

\begin{tabular}{|c|c|c|c|}
\hline Variable & $<65$ años $(\mathrm{n}=78)$ & $>65$ años $(n=40)$ & $\mathrm{p}$ \\
\hline Edad (años) ${ }^{*}$ & $45 \pm 12.81$ & $72 \pm 6.45$ & 0.000 \\
\hline SAPS II (puntos) ${ }^{*}$ & $66 \pm 12.5$ & $71 \pm 10.3$ & 0.001 \\
\hline Comorbilidades (\%) & & & 0.330 \\
\hline Diabetes & 28.4 & 37.7 & \\
\hline Hipertensión & 25.93 & 34.43 & \\
\hline Cardiopatías & 2.1 & 3.2 & \\
\hline Hematooncológicas & 4.93 & 6.57 & \\
\hline Origen de la infección (\%) & & & 0.220 \\
\hline Pulmonar & 32.0 & 20.0 & \\
\hline Abdominal & 39.7 & 62.5 & \\
\hline Urinaria & 20.5 & 12.5 & \\
\hline Tipo de tratamiento (\%) & & & 0.820 \\
\hline Médico & 32.0 & 27.5 & \\
\hline Quirúrgico & 67.9 & 72.5 & \\
\hline Tipo de infección (\%) & & & 0.190 \\
\hline Comunitaria & 71.8 & 60.0 & \\
\hline Nosocomial & 28.2 & 40.0 & \\
\hline No estancia en la UCI (días) ${ }^{*}$ & $6.74 \pm 5.96$ & $4.85 \pm 3.3$ & 0.060 \\
\hline Estancia en la UCI (días) ${ }^{*}$ & $5.19 \pm 4.0$ & $3.98 \pm 2.9$ & 0.640 \\
\hline Índice de choque modificado* & $1.45 \pm 0.49$ & $1.61 \pm 0.61$ & 0.120 \\
\hline $\mathrm{SvcO}_{2}(\%)^{*}$ & $68.75 \pm 12.58$ & $68.62 \pm 12.74$ & 0.950 \\
\hline$\Delta \mathrm{p}(\mathrm{v}-\mathrm{a}) \mathrm{CO}_{2} / \Delta \mathrm{p}(\mathrm{a}-\mathrm{v}) \mathrm{O}_{2}(\mathrm{mmHg} / \mathrm{mL})^{*}$ & $2.29 \pm 4.46$ & $1.91 \pm 1.62$ & 0.600 \\
\hline Norepinefrina $(\mu \mathrm{g} / \mathrm{kg} / \mathrm{min})^{*}$ & $0.29 \pm 0.18$ & $0.30 \pm 0.18$ & 0.600 \\
\hline Balance de líquidos $(\mathrm{mL})^{*}$ & $2,724 \pm 1,741$ & $2,768 \pm 1,669$ & 0.890 \\
\hline TRRLC (\%) & 42.3 & 57.5 & 0.110 \\
\hline Mortalidad (\%) & 36.0 & 67.5 & 0.001 \\
\hline
\end{tabular}

Abreviaturas: SAPS II = Simplified Acute Physiology Score II, UCI = Unidad de Cuidados Intensivos, $\mathrm{SvcO}_{2}=$ saturación venosa central de oxígeno, $\Delta \mathrm{p}(\mathrm{v}-\mathrm{a}) \mathrm{CO}_{2}=$ delta de presión venoarterial de dióxido de carbono, $\Delta \mathrm{p}(\mathrm{a}-\mathrm{v}) \mathrm{O}_{2}=$ delta de presión arteriovenosa de oxígeno, $\mathrm{BNP}=\mathrm{B}$-type Natriuretic $\mathrm{Peptide}_{\mathrm{PaO}} / \mathrm{FiO} \mathrm{O}_{2}=$ presión arterial de oxígeno/fracción inspiratoria de oxígeno, $\mathrm{HCO}_{3}{ }^{-}=$bicarbonato, $\mathrm{TRRLC}=$ terapia de reemplazo renal lenta continua.

* Media \pm desviación estándar. 
Tabla 2: Análisis bivariado entre los grupos vivo y no vivo en > 65 años de edad.

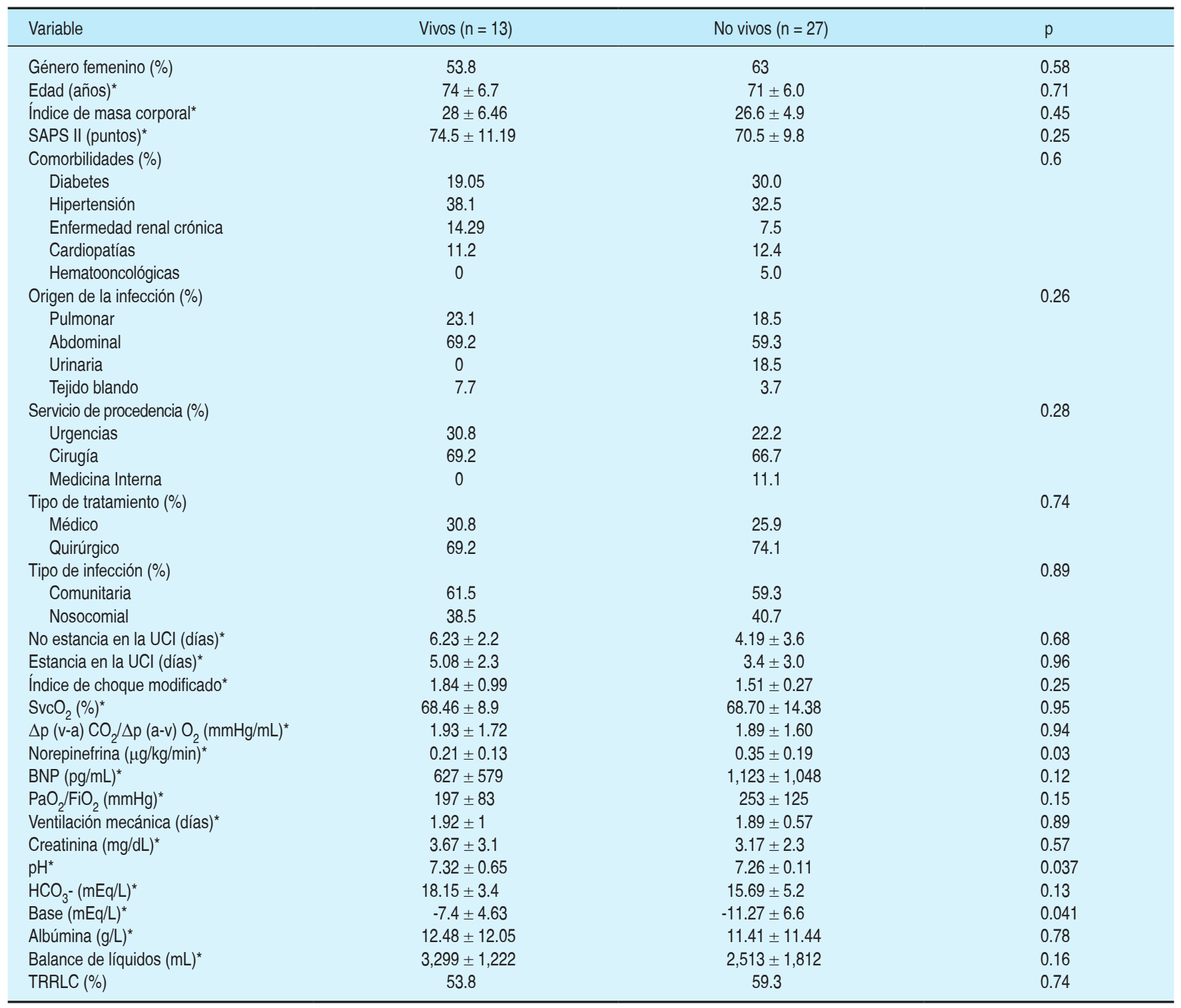

Abreviaturas: SAPS II = Simplified Acute Physiology Score II, UCl = Unidad de Cuidados Intensivos, $\mathrm{SvcO}_{2}=$ saturación venosa central de oxígeno, $\Delta \mathrm{p}(\mathrm{v}-\mathrm{a}) \mathrm{CO}_{2}=$ delta de presión venoarterial de dióxido de carbono, $\Delta \mathrm{p}(\mathrm{a}-\mathrm{v}) \mathrm{O}_{2}=$ delta de presión arteriovenosa de oxígeno, $\mathrm{BNP}=\mathrm{B}$-type Natriuretic Peptide, $\mathrm{PaO}_{2} / \mathrm{FiO}_{2}=$ presión arterial de oxígeno/fracción inspiratoria de oxígeno, $\mathrm{HCO}_{3}^{-}=$bicarbonato, $\mathrm{TRRLC}=$ terapia de reemplazo renal lenta continua.

* Media \pm desviación estándar.

esta misma población de pacientes al alta hospitalaria (a un año), encontrando un incremento que oscila entre 3 y $43 \% .{ }^{29} \mathrm{El}$ impacto de la edad por sí sola como factor de riesgo independiente para presentar una mayor mortalidad en los pacientes $>65$ años con choque séptico se observa uniformemente en las investigaciones epidemiológicas. ${ }^{30}$ Por ello, es importante conocer los factores que influyen en el pronóstico de esta población de pacientes especialmente vulnerables, con objetivo de orientar las decisiones de tratamiento y evaluar el pronóstico, lo anterior tomando en cuenta que existe suficiente evidencia sobre la asociación entre la intensidad del tratamiento y la supervivencia, incluso en el adulto mayor. ${ }^{31}$
La severidad de la enfermedad es uno de los factores más importantes que determinan la sobrevida en la UCI. Las diferentes escalas que predicen resultados tienen un incremento lineal con la edad y el riesgo de muerte. ${ }^{32}$ Chen y su grupo de investigación documentaron que una escala de APACHE II $\geq 25$ se considera como un factor de riesgo independiente para mortalidad en los pacientes $>65$ años que ingresan con diagnóstico de choque séptico $(<0.001) .{ }^{33}$ Asimismo, se ha documentado que la mortalidad en la $\mathrm{UCl}$ está relacionada significativamente con el fallo de órganos, ${ }^{34}$ razón por la cual las escalas de gravedad (evaluación de fallas orgánicas) mantienen una línea paralela con malos resulta- 
dos. Nuestra población de pacientes $>65$ años tuvieron media de SAPS II de 71 puntos, y los $<65$ años, una media de 66 puntos (con $p=0.001$ ), números que no difieren de la literatura ya descrita. Además, al evaluar a los pacientes $>65$ años en vivos y no vivos, documentamos que esta tendencia se mantiene, aunque sin diferencia significativa $(p=0.25)$. La dosis de norepinefrina no tuvo relevancia estadística con respecto a la edad, pero cuando se comparó en los mayores de 65 años entre vivos y no vivos $(0.21$ versus $0.35 \mu \mathrm{g} / \mathrm{kg} / \mathrm{min}$, respectivamente) sí existió relevancia estadística $(p=$ 0.03 ). Sabemos bien que cuando la hipotensión persiste y son necesarias dosis elevadas de vasopresores, el pronóstico suele ser malo, con una mortalidad superior al $50 \%$ a los 30 días. ${ }^{35}$ Otro dato interesante, aunque sin relevancia estadística $(p=0.03)$, fue el tiempo de estancia en la $\mathrm{UCl}$, el cual fue superior en los pacientes $<65$ años (media 5.19 días) respecto a los $>65$ años (media 3.98 días), esto probablemente porque se asocia con la mayor gravedad y muerte más temprana en estos pacientes, y no propiamente con una mejor evolución. Al evaluar sólo a los $>65$ años, el grupo de vivos tuvo mayor estancia en la $\mathrm{UCl}$, con una media de 5.08 días respecto al grupo de no vivos, el cual tuvo media de 3.4 días, aunque sin relevancia estadística. Los ancianos tienen un mayor riesgo de dependencia tras su ingreso a la $\mathrm{UCl}$ comparado con los pacientes más jóvenes, lo que condiciona una estancia más prolongada. ${ }^{36}$

La incidencia de comorbilidades contribuye al mal pronóstico. En comparación con pacientes más jóvenes, los > 65 años están más propensos a tener comorbilidades subyacentes. ${ }^{37}$ Por ejemplo, nosotros documentamos una mayor incidencia de comorbilidades como DM2, HAS, ERC, cardiopatía y hematooncológicos en los pacientes mayores de 65 años. Las cardiopatías en los pacientes $>65$ años respecto a los más jóvenes sí presentan diferencia estadística $(p=0.04)$. De hecho, los pacientes con insuficiencia cardiaca crónica presentan una mayor mortalidad en el con respecto al choque séptico, siendo identificada en algunos estudios como factor de riesgo independiente. ${ }^{38}$ Martin y otros reportaron que la insuficiencia cardiaca crónica era la morbilidad más frecuente en los pacientes $>$ 65 años con sepsis, cuya incidencia fue de hasta de $23.7 \%$, seguida de la DM2 (19.1\%) y de la enfermedad coronaria (18.1\%). ${ }^{39}$ Cuando evaluamos a los pacientes $>65$ años en vivos y no vivos, identificamos diferencia del BNP con una media de $627 \mathrm{pg} / \mathrm{mL}$ y $1,123 \mathrm{pg} / \mathrm{mL}$, respectivamente. Esto vuelve evidente que los valores sanguíneos de BNP poseen una capacidad pronóstica importante una vez determinado su valor en el momento del ingreso. Un patrón favorable sería el descenso desde el ingreso al momento del alta. ${ }^{40}$ En nuestro estudio, el origen de la infección más frecuente fue el foco abdominal, tanto en los pacientes < 65 años (39.7\%) como en los $>65$ años (62.5\%), con una diferencia estadísticamente significativa $(p=0.04)$. Otro estudio que incluyó a 237 pacientes $>65$ años y a $281<65$ años (n $=518$ ) documentó el foco abdominal como el más común con $52 \%$ en comparación con $36 \%(p=0.01)$ para ambos. ${ }^{41}$ Las complicaciones y la mortalidad postoperatorias se incrementan en pacientes ancianos sometidos a cirugía, y no es poco común el choque séptico como condicionante del evento quirúrgico. ${ }^{42,43} \mathrm{Al}$ respecto, nosotros registramos que $67.5 \%$ de los pacientes $>65$ años ingresó a la UCI después de realizarle una cirugía y sólo $43.6 \%$ en la población de pacientes $<65$ años. Además, los pacientes > 65 años tienen una mayor tasa de discapacidad prolongada después de la cirugía. Nuestros resultados apoyan a lo documentado en la literatura mundial.

Los pacientes con choque séptico a menudo requieren ventilación mecánica. Este soporte en los pacientes ancianos se asocia de forma independiente con una mayor mortalidad. ${ }^{44}$ Los pacientes $>65$ años tuvieron una media en los días de ventilación mecánica de 1.9, al igual que los $<65$ años, con $p=0.9$. En nuestra consideración, la menor cantidad de días de ventilación mecánica respecto a la literatura mundial es la gran incidencia de pacientes quirúrgicos, los cuales son menos complejos para el retiro de la ventilación mecánica con respecto a los pacientes médicos. Finalmente, cuando analizamos a los mayores de 65 años en vivos y no vivos, encontramos acidosis metabólica más severa en los no vivos (base $-11.27 \mathrm{mEq} / \mathrm{L}$ ) con respecto a los vivos (base-7.4 mEq/L), lo anterior documentado con el $\mathrm{pH}$ y la base, las cuales tienen $\mathrm{p}=$ 0.037 y 0.041 , respectivamente. Sabemos bien que la base (déficit de base en este caso) medida al ingreso a la $\mathrm{UCl}$ es de utilidad pronóstica en el paciente críticamente enfermo (entre ellos el séptico), sus niveles séricos predicen la mortalidad a través de la puntuación en sí mismo. ${ }^{45}$

Al revisar las diferentes variables epidemiológicas, nos pudimos dar cuenta cuáles son las que influyen en los resultados de los pacientes $>65$ años con choque séptico. Dentro de las limitaciones del estudio, reconocemos la ausencia de variables importantes, las cuales podrían influir en los resultados del choque séptico independientemente de la edad. También es importante resaltar que las variables fueron consignadas durante el ingreso a la $\mathrm{UCl}$ y no realizamos un seguimiento a los pacientes después del alta. Además, consideramos que al ser un estudio ambispectivo y realizado en un solo centro hospitalario se convierte en una limitante. Debemos considerar la gran cantidad de pacientes > 65 años que no ingresan a la $\mathrm{UCI}$, tomando en cuenta que son pacientes graves, aunque se encuentren fuera de la unidad. 


\section{CONCLUSIONES}

Los pacientes $>65$ años de edad que ingresan a la $\mathrm{UCl}$ tienen mayor gravedad respecto a pacientes más jóvenes, lo que condiciona una mayor mortalidad. Asimismo, en comparación con los pacientes $<65$ años, están más propensos a tener comorbilidades subyacentes. Dentro de las comorbilidades, la diabetes mellitus tipo 2 es más frecuente. La etiología abdominal es la causa más común de choque séptico, y la acidosis metabólica grave es un factor de riesgo para mortalidad en los pacientes $>65$ años.

\section{BIBLIOGRAFÍA}

1. Marik PE. Management of the critically ill geriatric patient. Crit Care Med. 2006;34:S176-S182.

2. Population Division, Department of Economic and Social Affairs. World Population Ageing: 1950-2050; 2001. Disponible en: http://www.un.org/esa/population/publications/ worldageing19502050.

3. Padkin A, Goldfrad C, Brady AR, Young D, Black N, Rowan $\mathrm{K}$. Epidemiology of severe sepsis occurring in the first $24 \mathrm{~h}$ in intensive care units in England, Wales, and Northern Ireland. Crit Care Med. 2003;31:2232-2238.

4. Dombrovskiy VY, Martin AA, Sunderram J, Paz HL. Rapid increase in hospitalization and mortality rates for severe sepsis in the United States: A trend analysis from 1993 to 2003. Crit Care Med. 2007;35:1244-1250.

5. Angus DC, Kelley MA, Schmitz RJ, White A, Popovich J. Caring for the critically ill patient. Current and projected workforce requirements for care of the critically ill and patients with pulmonary disease: can we meet the requirements of an aging population? JAMA. 2000;284:2762-2770.

6. Martin CM, Priestap F, Fisher H, Fowler RA, Heyland DK, Keenan $\mathrm{SP}$, et al. A prospective, observational registry of patients with severe sepsis: the Canadian Sepsis Treatment and Response Registry. Crit Care Med. 2009;37:81-88.

7. Martin GS, Mannino DM, Moss M. The effect of age on the development and outcome of adult sepsis. Crit Care Med. 2006;34:15-21.

8. Prescott $\mathrm{HC}$, Angus DC. Enhancing recovery from sepsis: a review. JAMA. 2018;319(1):62-75.

9. Weycker D, Akhras KS, Edelsberg J, Angus D, Oster G. Longterm mortality and medical care charges in patients with severe sepsis. Crit Care Med. 2003;31:2316-2323.

10. Braun L, Riedel AA, Cooper LM. Severe sepsis in managed care: analysis of incidence, one-year mortality, and associated costs of care. J Manag Care Pharm. 2004;10:521-530.

11. Caterino JM. Evaluation and management of geriatric infections in the emergency department. Emerg Med Clin North Am. 2008;26:319-343.

12. Holloszy JO. The biology of aging. Mayo Clin Proc. 2000;75(Suppl):S3-S8.

13. Martin GS, Mannino DM, Moss M. Effect of age on the development and outcome with sepsis. Am J Respir Crit Care Med. 2003;167:A837.

14. Bone RC, Balk RA, Cerra FB, Dellinger RP, Fein AM, Knaus WA, et al. Definitions for sepsis and organ failure and guidelines for the use of innovative therapies in sepsis. The ACCP/ SCCM Consensus Conference Committee. American College of Chest Physicians/Society of Critical Care Medicine. Chest. 1992;101:1644-1655.

15. Gleckman R, Hibert D. Afebrile bacteremia: a phenomenon in geriatric patients. JAMA. 1982;248:1478-1481.

16. Rajagopalan S, Yoshikawa TT. Antimicrobial therapy in the elderly. Med Clin North Am. 2001;85:133-147.

17. Yoshikawa T. Epidemiology and unique aspects of aging and infectious diseases. Clin Infect Dis. 2000;30:931-933.
18. Balaban RS, Nemoto S, Finkel T. Mitochondria, oxidants, and aging. Cell. 2005;120:483-495.

19. Lakatta EG. Age-associated cardiovascular changes in health impact on cardiovascular disease in older persons. Heart Fail Rev. 2002;7:29-49.

20. Morley JE, Reese SS. Clinical implications of the aging heart. Am J Med. 1989;86:77-86.

21. Zeleznik J. Normative aging of the respiratory system. Clin Geriatr Med. 2003;19:1-18.

22. Cockcroft DW, Gault MH. Prediction of creatinine clearance from serum creatinine. Nephron. 1976;16:31-34.

23. Peerless JR, Epstein CD, Martin JE, Pinchak AC, Malangoni MA. Oxygen consumption in the early postinjury period: Use of continuous, on-line indirect calorimetry. Crit Care Med. 2000;28:395-340.

24. Fabbri A, Marchesini G, Benazzi B, Morelli A, Montesi D, Bini $C$, et al. Old subjects with sepsis in the emergency department: trend analysis of case fatality rate. BMC Geriatr. 2019;19(1):372. doi: 10.1186/s12877-019-1384-8.

25. Angus DC, Linde-Zwirble WT, Lidicker J, Clermont G, Carcillo J, Pinsky MR. Epidemiology of severe sepsis in the United States: analysis of incidence, outcome, and associated costs of care. Crit Care Med. 2001;29:1303-1310.

26. Rivlin M. Should age based rationing of health care be illegal? BMJ. 1999;319:1379.

27. Sinuff T, Kahnamoui K, Cook DJ, Luce JM, Levy MM. Rationing critical care beds: a systematic review. Crit Care Med. 2004;32:1588-1597.

28. Nasa P, Juneja D, Singh O, et al. Severe sepsis and its impact on outcome in elderly and very elderly patients admitted in intensive care unit. J Intensive Care Med. 2012;27(3):179-183.

29. Yende S, Angus DC. Long-term outcomes from sepsis. Curr Infect Dis Rep. 2007;9:382-386.

30. Lemay AC, Anzueto A, Restrepo MI, Mortensen EM. Predictors of long-term mortality after severe sepsis in the elderly. Am J Med Sci. 2014;347(4):282-288.

31. Lerolle N, Trinquart L, Bornstain C, Tadié JM, Imbert A, Diehl JL, et al. Increased intensity of treatment and decreased mortality in elderly patients in an intensive care unit over a decade. Crit Care Med. 2010;38:59-64.

32. Mayer-Oakes SA, Oye RK, Leake B. Predictors of mortality in older patients following medical intensive care: the importance of functional status. J Am Geriatr Soc. 1991;39:862-868.

33. Chen $\mathrm{CM}$, Cheng KC, Chan KS, Yu WL. Age may not influence the outcome of patients with severe sepsis in intensive care units. Int J Gerontol. 2014;8(1):22-26.

34. Opal SM, Girard TD, Ely EW. The immunopathogenesis of sepsis in elderly patients. Clin Infect Dis. 2005:41:S504-S512.

35. Khanna A, English SW, Wang XS, Ham K, Tumlin J, Szerlip H, et al. Angiotensin II for the treatment of vasodilatory shock. $N$ Engl $J$ Med. 2017;377(5):419-430.

36. Nfor TK, Walsh TS, Prescott RJ. The impact of organ failures and their relationship with outcome in intensive care: analysis of a prospective multicentre database of adult admissions. Anaesthesia. 2006;61:731-738.

37. Rellos K, Falagas ME, Vardakas KZ, Sermaides G, Michalopoulos A. Outcome of critically ill oldest-old patients (aged 90 and older) admitted to the Intensive Care Unit. J Am Geriatr Soc. 2006;54(1):110-114

38. Brun-Buisson C, Meshaka P, Pinton P, Vallet B. EPISEPSIS: a reappraisal of the epidemiology and outcome of severe sepsis in French intensive care units. Intensive Care Med. 2004;30:580-588

39. Martin-Loeches I, Guia MC, Vallecoccia MS, Suarez D, Ibarz $M$, Irazabal M, et al. Risk factors for mortality in elderly and very elderly critically ill patients with sepsis: a prospective, observational, multicenter cohort study. Ann Intensive Care. 2019;9(1):26.

40. Almenar L, Martínez-Dolz L. Péptidos natriuréticos en insuficiencia cardiaca. Rev Esp Cardiol Supl. 2006;6(6):15F-26F.

41. Carbajal-Guerrero J, Cayuela-Domínguez A, Fernández-García E, Aldabó-Pallás T, Márquez-Vácaro JA, Ortiz-Leyba C, et al. 
Epidemiología y pronóstico tardío de la sepsis en ancianos. Med Intensiva. 2014;38(1):21-32.

42. Barlow AP, Zarifa Z, Shillito RG, et al. Surgery in a geriatric population. Ann R Coll Surg Engl. 1989;71:110-114.

43. Liu LL, Leung JM. Predicting adverse postoperative outcomes in patients aged 80 years or older. J Am Geriatr Soc. 2000;48:405-412.

44. Nierman DM, Schechter CB, Cannon LM, Meier DE. Outcome prediction model for very elderly critically ill patients. Crit Care Med. 2001;29:1853-1859.

45. Hajjar LA, Nakamura RE, de Almeida JP, et al. Lactate and base deficit are predictors of mortality in critically ill patients with cancer. Clinics. 2011;66(12):2037-2042.
Patrocinios y relación de conflictos de interés: el presente trabajo no recibió patrocinios, sólo se utilizaron recursos propios de nuestra Unidad de Cuidados Intensivos. Los autores declaramos no tener conflictos de interés.

Correspondencia:

Dr. Jesús Salvador Sánchez Díaz

Av. Cuauhtémoc S/N, Col. Formando Hogar, 91897, Veracruz, Veracruz.

Teléfono: 2299342031

E-mail: drsalvadorsanchezdiaz@gmail.com 